\title{
Upper semicontinuity of pullback attractors for a nonautonomous damped wave equation
}

\author{
Yonghai Wang ${ }^{1,2^{*}}$ (D), Minhui $H u^{1}$ and Yuming Qin ${ }^{1,2}$
}

\author{
"Correspondence: \\ yonghai@dhu.edu.cn \\ ${ }^{1}$ College of Science, Donghua \\ University, Shanghai, 201620, China \\ ${ }^{2}$ Institute for Nonlinear Sciences, \\ Donghua University, Shanghai, \\ 201620, China
}

\begin{abstract}
In this paper, we study the local uniformly upper semicontinuity of pullback attractors for a strongly damped wave equation. In particular, under some proper assumptions, we prove that the pullback attractor $\left\{A_{\varepsilon}(t)\right\}_{t \in \mathbb{R}}$ of Eq. (1.1) with $\varepsilon \in[0,1]$ satisfies $\lim _{\varepsilon \rightarrow \varepsilon_{0}} \sup _{t \in[a, b]} \operatorname{dist}_{H_{0}^{1} \times L^{2}}\left(A_{\varepsilon}(t), A_{\varepsilon_{0}}(t)\right)=0$ for any $[a, b] \subset \mathbb{R}$ and $\varepsilon_{0} \in[0,1]$.

MSC: 37L05; 35B40; 35B41
\end{abstract}

Keywords: Pullback attractor; Upper semicontinuity; Wave equation

\section{Introduction}

The theory of pullback (or random) attractors is a useful tool to study the long-time behavior of nonautonomous (or random) dynamical systems (see [1, 3, 7] and references therein), in which the trajectory can be unbounded as "time" goes to infinity, and thus classical theory of global (or uniform) attractors is not applicable. A pullback attractor is a parameterized family $\{\mathscr{A}(t)\}_{t \in \mathbb{R}}$ of nonempty compact sets of the state space, which attracts bounded deterministic sets starting from earlier time. In recent years the upper semicontinuity of pullback attractors for dynamical systems with different kind of perturbations has also been widely studied (see, e.g., [2, 6, 10, 13, 14, 19, 21, 23, 24]). Simply speaking, if $\left\{A_{\varepsilon}(t)\right\}_{t \in \mathbb{R}}$ is the pullback attractor generated by the perturbed dynamical systems and $\left\{A_{0}(t)\right\}_{t \in \mathbb{R}}$ is the pullback attractor for the unperturbed one, then we say that $\left\{A_{\varepsilon}(t)\right\}_{t \in \mathbb{R}}$ and $\left\{A_{0}(t)\right\}_{t \in \mathbb{R}}$ are upper semicontinuous in a metric space $(X, d)$ if

$$
\lim _{\varepsilon \rightarrow 0} \operatorname{dist}_{X}\left(A_{\varepsilon}(t), A_{0}(t)\right)=0, \quad \forall t \in \mathbb{R}
$$

where, $\operatorname{dist}_{X}(\cdot, \cdot)$ denotes the Hausdorff semidistance $\operatorname{dist}_{X}(A, B)=\sup _{a \in A} \inf _{b \in B} d(a, b)$.

(c) The Author(s) 2021. This article is licensed under a Creative Commons Attribution 4.0 International License, which permits use sharing, adaptation, distribution and reproduction in any medium or format, as long as you give appropriate credit to the original author(s) and the source, provide a link to the Creative Commons licence, and indicate if changes were made. The images or other third party material in this article are included in the article's Creative Commons licence, unless indicated otherwise in a credit line to the material. If material is not included in the article's Creative Commons licence and your intended use is not permitted by statutory regulation or exceeds the permitted use, you will need to obtain permission directly from the copyright holder. To view a copy of this licence, visit http://creativecommons.org/licenses/by/4.0/. 
In this paper, we consider the upper semicontinuity of pullback attractors for the following strongly damped wave equation:

$$
\left\{\begin{array}{l}
\partial_{t}^{2} u+\partial_{t} u-\varepsilon \Delta \partial_{t} u-\Delta u+f(u)=g(x, t) \quad \text { in } \Omega \times[r, \infty), \\
\left(u(r), \partial_{t} u(r)\right)=\left(u_{r}, u_{r}^{\prime}\right), \\
\left.u(x, t)\right|_{\partial \Omega \times[r, \infty)}=0,
\end{array}\right.
$$

where $\Omega \subset \mathbb{R}^{3}$ ia a bounded smooth domain, and $\varepsilon \in[0,1]$. For the nonlinearity $f \in C^{2}(\mathbb{R})$ with $f(0)=0$, we assume that it satisfies:

$$
\begin{aligned}
& \left|f^{\prime \prime}(u)\right| \leq C(|u|+1), \\
& \liminf _{|u| \rightarrow \infty} f^{\prime}(u)>-\lambda_{1},
\end{aligned}
$$

where $\lambda_{1}>0$ is the first eigenvalue of $-\Delta$.

The external force $g(x, t)$ is assumed to satisfy: $g(x, t), \partial_{t} g(x, t) \in L_{\text {loc }}^{2}\left(\mathbb{R} ; L^{2}(\Omega)\right)$, and

$$
\int_{-\infty}^{t} e^{\sigma s}\|g(x, s)\|^{2} d s<\infty \quad \text { for all } t \in \mathbb{R}
$$

where the positive constant $\sigma$ will be settled in the proof of Lemma 3.1.

The dynamic behavior of analogous equations have been analyzed in the literature under different hypotheses. In the autonomous case (i.e., the forcing term $g(x, t)=g(x)$ ), the wellposedness, existence, and regularity of global attractors have been studied extensively for more general damped wave equations $[4,5,8,9,12,15,16]$, and the exponential attractors and dimension estimates for global attractors are considered in $[11,15,17]$. In the nonautonomous case, we refer the readers to $[20-22,25]$ and references therein.

When $\varepsilon=0$, Eq. (1.1) reduces to the usual wave equation without strong damping term $-\Delta \partial_{t} u$. Our main purpose in this paper is to study the limiting behavior of Eq. (1.1) as $\varepsilon$ goes to 0 . More precisely, we will prove the upper semicontinuity of pullback attractors in $H_{0}^{1}(\Omega) \times L^{2}(\Omega)$ for Eq. (1.1), that is, that the pullback attractor $\left\{A_{\varepsilon}(t)\right\}_{t \in \mathbb{R}}(\varepsilon \in[0,1])$ for Eq. (1.1) satisfies

$$
\lim _{\varepsilon \rightarrow \varepsilon_{0}} \sup _{t \in[a, b]} \operatorname{dist}_{H_{0}^{1} \times L^{2}}\left(A_{\varepsilon}(t), A_{\varepsilon_{0}}(t)\right)=0 \quad \text { for all } \varepsilon_{0} \in[0,1] \text { and }[a, b] \subset \mathbb{R} .
$$

For Eq. (1.1), if the initial data belong to $H_{0}^{1}(\Omega) \times L^{2}(\Omega)$, then its solution is always in $H_{0}^{1}(\Omega) \times L^{2}(\Omega)$ and has no higher regularity, and we cannot obtain the compactness property by showing the boundedness of solutions in higher regular phase spaces. In this paper, we apply the techniques of Zelik [25] to overcome this difficulty and establish the asymptotic compactness of solution operators with perturbations (see Lemmas 3.3-3.5).

The structure of the paper is as follows. In the next section, we first recall some basic concepts and conclusions of the theory of pullback attractors and then prove an abstract result for verifying the upper semicontinuity of pullback attractors (Theorem 2.2), by applying which we prove the upper semicontinuity of pullback attractors for Eq. (1.1) in Sect. 3.

We introduce some notation that will be used in the paper. We denote by $\langle\cdot, \cdot \cdot\rangle$ and $\|\cdot\|$ the inner product and norm in $L^{2}(\Omega)$, respectively. Let $\mathcal{H}^{\alpha}=D\left((-\Delta)^{\frac{\alpha}{2}}\right)(\alpha \in \mathbb{R})$ be the 
scale of Hilbert spaces generated by the Laplacian with Dirichlet boundary conditions on $L^{2}(\Omega)$ (see [18] for more detail) and endowed with standard inner products and norms, respectively,

$$
\langle\cdot, \cdot\rangle_{\mathcal{H}^{\alpha}}=\left\langle(-\Delta)^{\frac{\alpha}{2}} \cdot,(-\Delta)^{\frac{\alpha}{2}} \cdot\right\rangle \text { and }\|\cdot\|_{\mathcal{H}^{\alpha}}=\left\|(-\Delta)^{\frac{\alpha}{2}} \cdot\right\| .
$$

In particular,

$$
\mathcal{H}^{-1}=H^{-1}(\Omega), \quad \mathcal{H}^{0}=L^{2}(\Omega), \quad \mathcal{H}^{1}=H_{0}^{1}(\Omega) .
$$

Then we have the continuous embeddings $\mathcal{H}^{s} \hookrightarrow \mathcal{H}^{r}$ for any $s>r$,

$$
\mathcal{H}^{s} \hookrightarrow L^{\frac{6}{3-2 s}}(\Omega), \quad \forall s \in\left[0, \frac{3}{2}\right)
$$

and the following inequalities.

Interpolation inequalities: if $r=\theta s+(1-\theta) q$, where $r, s, q \in \mathbb{R}, s \geq q$, and $\theta \in[0,1]$, then there exists a constant $C>0$ such that

$$
\|u\|_{\mathcal{H}^{r}} \leq C\|u\|_{\mathcal{H}^{s}}^{\theta}\|u\|_{\mathcal{H}^{q}}^{1-\theta}, \quad \forall u \in \mathcal{H}^{s}
$$

Generalized Poincaré inequality:

$$
\lambda_{1}\|u\|_{\mathcal{H}^{\alpha}}^{2} \leq\|u\|_{\mathcal{H}^{\alpha+1}}^{2}, \quad \forall u \in \mathcal{H}^{\alpha+1}
$$

We define the product Hilbert spaces as follows:

$$
\mathcal{E}^{-1}=L^{2}(\Omega) \times H^{-1}(\Omega), \quad \mathcal{E}=H_{0}^{1}(\Omega) \times L^{2}(\Omega),
$$

and

$$
\mathcal{E}^{\alpha}=\mathcal{H}^{\alpha+1} \times \mathcal{H}^{\alpha}(\alpha \in \mathbb{R})
$$

For any given function $u(t)$, we shortly write

$$
\xi_{u}(t)=\left(u(t), \partial_{t} u(t)\right) \quad \text { and } \quad\left\|\xi_{u}(t)\right\|_{\mathcal{E}}^{2}=\|\nabla u\|^{2}+\left\|\partial_{t} u\right\|^{2} .
$$

Throughout the paper, the symbols $C$ and $Q$ stand for a generic positive constant and a generic positive increasing function, respectively.

\section{Preliminaries}

In this section, we collect some basic facts from general theory of pullback attractors (see, e.g., $[1,3])$ and then state an abstract result for verifying the upper semicontinuity of pullback attractors.

Let us define a nonautonomous dynamical system by a process on a Banach space $X$ with norm $\|\cdot\|_{X}$, that is, a family of continuous mappings $U(t, \tau): X \rightarrow X, t \geq \tau$, such that $U(\tau, \tau)=\operatorname{Id}$ and $U(t, s) U(s, \tau)=U(t, \tau)$ for all $t \geq s \geq \tau$. 
Definition 2.1 A family of compact sets $\mathscr{A}=\{A(t)\}_{t \in \mathbb{R}}$ is called a pullback attractor for process $U(\cdot, \cdot)$ if

(i) $\mathscr{A}$ is pullback attracting, that is, $\lim _{\tau \rightarrow \infty} \operatorname{dist}_{X}(U(t, t-\tau) D, A(t))=0$ for all bounded $D \subset X$

(ii) $\mathscr{A}$ is invariant, that is, $U(t, \tau) A(\tau)=A(t)$ for all $t \geq \tau$.

Definition 2.2 A family of sets $\mathscr{D}=\{D(t)\}_{t \in \mathbb{R}}$ is said to be pullback absorbing with respect to $U(\cdot, \cdot)$ if for every $t \in \mathbb{R}$ and any bounded $D \subset X$, there exists $T>0$ (which depends on $t$ and $D$ ) such that

$$
U(t, t-\tau) D \subset D(t) \quad \text { for all } \tau \geq T \text {. }
$$

Definition 2.3 A process $U(\cdot, \cdot)$ is said to be pullback $\mathscr{D}$-asymptotically compact in $X$ if for any $t \in \mathbb{R}$ and any sequences $\tau_{n} \stackrel{n \rightarrow \infty}{\longrightarrow} \infty$ and $x_{n} \in D\left(t-\tau_{n}\right)$, the sequence $\left\{U\left(t, t-\tau_{n}\right) x_{n}\right\}_{n \in \mathbb{N}}$ is relatively compact in $X$.

Theorem 2.1 (see [3]) Let a family $\mathscr{D}=\{D(t)\}_{t \in \mathbb{R}}$ be pullback absorbing, and let $U(\cdot, \cdot)$ be pullback $\mathscr{D}$-asymptotically compact in $X$. Then the family $\mathscr{A}=\{A(t)\}_{t \in \mathbb{R}}$ defined by $A(t):=\Lambda(\mathscr{D}, t)$, where

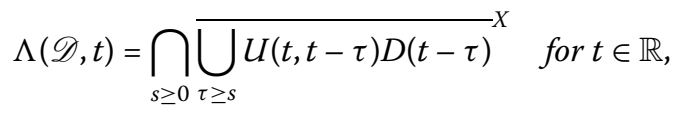

is a pullback attractor for $U(\cdot, \cdot)$. Moreover, if for any $t \in \mathbb{R}$, there exists $T>0$ (which depends on $t$ ) such that

$$
U(t, t-\tau) D(t-\tau) \subset D(t) \quad \text { for all } \tau \geq T
$$

then

$$
\lim _{\tau \rightarrow \infty} \operatorname{dist}_{X}(U(t, t-\tau) D(t-\tau), A(t))=0 \text { for each } t \in \mathbb{R} .
$$

Lemma 2.1 Assume that for every $\varepsilon \in[0, \mu]$, a process $U_{\varepsilon}(\cdot, \cdot)$ has a pullback absorbing family $\mathscr{D}_{\varepsilon}=\left\{D_{\varepsilon}(t)\right\}_{t \in \mathbb{R}}$ satisfying (2.2). Assume that for any sequences $\left\{\varepsilon_{n}\right\}_{n \in \mathbb{N}} \subset[0, \mu]$, $\left\{t_{n}\right\}_{n \in \mathbb{N}} \subset[a, b] \subset \mathbb{R},\left\{\tau_{n}\right\}_{n \in \mathbb{N}} \subset \mathbb{R}^{+}$with $\tau_{n} \stackrel{n \rightarrow \infty}{\longrightarrow} \infty$, and $x_{n} \in D_{\varepsilon_{n}}\left(a-\tau_{n}\right)$, the sequence $\left\{U_{\varepsilon_{n}}\left(t_{n}, a-\tau_{n}\right) x_{n}\right\}_{n \in \mathbb{N}}$ is precompact in $X$. Then

(i) for every $\varepsilon \in[0, \mu], U_{\varepsilon}(\cdot, \cdot)$ has a pullback attractor $\mathscr{A}_{\varepsilon}=\left\{A_{\varepsilon}(t)\right\}_{t \in \mathbb{R}}$;

(ii) $\bigcup_{t \in[a, b]} \bigcup_{\varepsilon \in[0, \mu]} A_{\varepsilon}(t)$ is precompact in $X$.

Proof From Theorem 2.1 we immediately get (i). Taking any sequence $x_{n} \in \bigcup_{t \in[a, b]} \bigcup_{\varepsilon \in[0, \mu]} A_{\varepsilon}(t)$, without loss of generality, we let $x_{n} \in A_{\varepsilon_{n}}\left(t_{n}\right)$. Then we can find sequences $\tau_{n} \rightarrow \infty$ and $\xi_{n} \in D_{\varepsilon_{n}}\left(a-\tau_{n}\right)$ such that

$$
\left\|U_{\varepsilon_{n}}\left(t_{n}, a-\tau_{n}\right) \xi_{n}-x_{n}\right\| \leq \frac{1}{n}, \quad \forall n \in \mathbb{N} .
$$

Then from the assumptions we easily obtain the precompactness of $\left\{x_{n}\right\}_{n \mathbb{N}}$. 
The theory for verifying the upper semicontinuity of pullback attractors has been considered by many authors; see $[7,13]$ and references therein. By applying the ideas of $[13$, Theorem 4.1] we get the following result.

Theorem 2.2 Let $X, Y$ be two Banach spaces with norms $\|\cdot\|_{X}$ and $\|\cdot\|_{Y}$, respectively, and let $X$ be continuously embedded into $Y$. Assume that for every $\varepsilon \in[0, \mu]$, a process $U_{\varepsilon}(\cdot, \cdot)$ has a pullback absorbing family $\mathscr{D}_{\varepsilon}=\left\{D_{\varepsilon}(t)\right\}_{t \in \mathbb{R}}$ satisfying (2.2), and a pullback attractor $\mathscr{A}_{\varepsilon}=\left\{A_{\varepsilon}(t)\right\}_{t \in \mathbb{R}}$ is given by Theorem 2.1. Suppose the following assumptions hold:

(i) $\mathscr{D}_{\varepsilon}=\left\{D_{\varepsilon}(t)\right\}_{t \in \mathbb{R}}$ is independent of $\varepsilon$, and $D_{\varepsilon}(t)$ is closed in $X$ for each $t \in \mathbb{R}$;

(ii) for any $\tau>0$, any sequences $\left\{t_{n}\right\}_{n \in \mathbb{N}} \subset[a, b]$ such that $t_{n} \stackrel{n \rightarrow \infty}{\longrightarrow} t_{0},\left\{\varepsilon_{n}\right\}_{n \in \mathbb{N}} \subset[0, \mu]$ such that $\varepsilon_{n} \stackrel{n \rightarrow \infty}{\longrightarrow} \varepsilon_{0}$, and $\left\{x_{n}\right\}_{n \in \mathbb{N}} \subset X$ such that $x_{n} \stackrel{n \rightarrow \infty}{\longrightarrow} x_{0}$ in $X$,

$$
U_{\varepsilon_{n}}\left(t_{n}, a-\tau\right) x_{n} \stackrel{n \rightarrow \infty}{\longrightarrow} U_{\varepsilon_{0}}\left(t_{0}, a-\tau\right) x_{0} \quad \text { in } Y
$$

(iii) for any $[a, b] \subset \mathbb{R}$,

$$
\bigcup_{t \in[a, b]} \bigcup_{\varepsilon \in[0, \mu]} A_{\varepsilon}(t) \text { is precompact in } X .
$$

Then

$$
\lim _{\varepsilon \rightarrow \varepsilon_{0}} \sup _{t \in[a, b]} \operatorname{dist}_{X}\left(A_{\varepsilon}(t), A_{\varepsilon_{0}}(t)\right)=0 \text { for all } \varepsilon_{0} \in[0, \mu] .
$$

Proof Step 1. We prove that under our assumptions, for any $t \in \mathbb{R}$, any sequences $\left\{\varepsilon_{n}\right\}_{n \in \mathbb{N}} \subset[0, \mu]$ such that $\varepsilon_{n} \stackrel{n \rightarrow \infty}{\longrightarrow} \varepsilon_{0}$, and $y_{n} \in A_{\varepsilon_{n}}(t)$, there exist $y_{0} \in A_{\varepsilon_{0}}(t)$ and a subsequence $\left\{y_{n_{k}}\right\}_{k \in \mathbb{N}} \subset\left\{y_{n}\right\}_{n \in \mathbb{N}}$ such that

$$
y_{n_{k}} \stackrel{k \rightarrow \infty}{\longrightarrow} y_{0} \quad \text { in } X
$$

Let $\tau_{m} \stackrel{m \rightarrow \infty}{\longrightarrow} \infty$. For all $m, n \in \mathbb{N}$, there exist $z_{n}^{(m)} \in A_{\varepsilon_{n}}\left(a-\tau_{m}\right)$ such that

$$
y_{n}=U_{\varepsilon_{n}}\left(t, a-\tau_{m}\right) z_{n}^{(m)} .
$$

By assumption (i) we let

$$
D_{\varepsilon}(t)=D(t) \quad \text { for all } t \in \mathbb{R} \text { and } \varepsilon \in[0, \mu],
$$

and by assumption (iii), without loss of generality, for each $m$, we let

$$
z_{n}^{(m)} \stackrel{n \rightarrow \infty}{\longrightarrow} z_{m} \in D\left(a-\tau_{m}\right) .
$$

Let $y_{0, m}=U_{\varepsilon_{0}}\left(t, a-\tau_{m}\right) z_{m}$, and, without loss of generality, let

$$
y_{0, m} \stackrel{m \rightarrow \infty}{\longrightarrow} y_{0} \in A_{\varepsilon_{0}}(t) .
$$


By assumption (ii) we find

$$
\lim _{n \rightarrow \infty}\left\|y_{n}-y_{0, m}\right\|_{Y}=\lim _{n \rightarrow \infty}\left\|U_{\varepsilon_{n}}\left(t, a-\tau_{m}\right) z_{n}^{(m)}-U_{\varepsilon_{0}}\left(t, a-\tau_{m}\right) z_{m}\right\|_{Y}=0
$$

Then, combining (2.9) and (2.10), for any $\delta>0$, we can find $m_{\delta}, N_{\delta} \in \mathbb{N}$ such that

$$
\left\|y_{n}-y_{0}\right\|_{Y} \leq\left\|y_{n}-y_{0, m_{\delta}}\right\|_{Y}+\left\|y_{0, m_{\delta}}-y_{0}\right\|_{Y} \leq \delta \quad \text { for all } n \geq N_{\delta} \text {. }
$$

From assumption (iii) we also know that $\left\{y_{n}\right\}_{n \in \mathbb{N}}$ is precompact in $X$. Thus by (2.11), noting that $X \hookrightarrow Y$, we immediately get (2.7).

Step 2. We claim that for any sequences $\left\{t_{n}\right\}_{n \in \mathbb{N}} \subset[a, b]$ such that $t_{n} \stackrel{n \rightarrow \infty}{\longrightarrow} t_{0},\left\{\varepsilon_{n}\right\}_{n \in \mathbb{N}} \subset$ $[0, \mu]$ such that $\varepsilon_{n} \stackrel{n \rightarrow \infty}{\longrightarrow} \varepsilon_{0}$, and $x_{n} \in A_{\varepsilon_{n}}\left(t_{n}\right)$, there exist a subsequence $\left\{x_{n_{k}}\right\}_{k \in \mathbb{N}} \subset\left\{x_{n}\right\}_{n \in \mathbb{N}}$ and $x_{k}^{*} \in A_{\varepsilon_{0}}\left(t_{n_{k}}\right)$ such that for any $\delta>0$, we can find $N \in \mathbb{N}$ large enough such that

$$
\left\|x_{n_{k}}-x_{k}^{*}\right\|_{X} \leq \delta \quad \text { for all } k \geq N
$$

Let $\tau>0$. We can find $y_{n} \in A_{\varepsilon_{n}}(a-\tau)$ such that

$$
x_{n}=U_{\varepsilon_{n}}\left(t_{n}, a-\tau\right) y_{n} \quad \text { for all } n \in \mathbb{N} \text {. }
$$

By (2.7), without loss of generality, we let

$$
y_{n} \stackrel{n \rightarrow \infty}{\longrightarrow} y_{0}\left(\in A_{\varepsilon_{0}}(a-\tau)\right) .
$$

Setting

$$
x_{n}^{*}=U_{\varepsilon_{0}}\left(t_{n}, a-\tau\right) y_{0}\left(\in A_{\varepsilon_{0}}\left(t_{n}\right)\right)
$$

and using assumption (ii), we readily check that

$$
\lim _{n \rightarrow \infty}\left\|x_{n}-x_{n}^{*}\right\|_{Y}=0
$$

On the other hand, assumption (iii) implies that sequences $\left\{x_{n}\right\}_{n \in \mathbb{N}}$ and $\left\{x_{n}^{*}\right\}_{n \in \mathbb{N}}$ are precompact in $X$, from which, together with (2.14), noting that $X \hookrightarrow Y$, we immediately obtain (2.12).

Now we are ready to prove (2.6). If not true, then we can find $\delta>0$, sequences $\left\{\varepsilon_{n}\right\}_{n \in \mathbb{N}} \subset$ $[0, \mu]$ such that $\varepsilon_{n} \stackrel{n \rightarrow \infty}{\longrightarrow} \varepsilon_{0},\left\{t_{n}\right\}_{n \in \mathbb{N}} \subset[a, b]$ such that $t_{n} \stackrel{n \rightarrow \infty}{\longrightarrow} t_{0}$, and $x_{n} \in A_{\varepsilon_{n}}\left(t_{n}\right)$ such that

$$
\operatorname{dist}_{X}\left(x_{n}, A_{\varepsilon_{0}}\left(t_{n}\right)\right) \geq \delta \quad \text { for all } n \in \mathbb{N} \text {. }
$$

It follows from (2.12) that we can extract a subsequence $\left\{x_{n_{k}}\right\}_{k \in \mathbb{N}}$ from $\left\{x_{n}\right\}_{n \in \mathbb{N}}$ and $x_{k}^{*} \in$ $A_{\varepsilon_{0}}\left(t_{n_{k}}\right)$ such that

$$
\left\|x_{n_{k}}-x_{k}^{*}\right\|_{X} \stackrel{k \rightarrow \infty}{\longrightarrow} 0
$$

which contradicts (2.15). The proof is completed. 


\section{Upper semicontinuity of pullback attractors for Eq. (1.1)}

The existence and uniqueness of (weak) solutions $u$ to Eq. (1.1) is classical (see, e.g., [4, 5]) and can be obtained by the standard Faedo-Galerkin method. Such solutions satisfy: for any $\varepsilon \geq 0$ and $[r, T] \subset \mathbb{R}$,

$$
u \in C\left([r, T], H_{0}^{1}(\Omega)\right), \quad \partial_{t} u \in C\left([r, T], L^{2}(\Omega)\right) .
$$

As a consequence, for any $\varepsilon \geq 0$, we can construct the process $U_{\varepsilon}(t, r)$ associated with Eq. (1.1) as follows:

$$
U_{\varepsilon}(t, r) \xi=\left(u(t), \partial_{t} u(t)\right) \quad \text { with } \xi \in \mathcal{E}
$$

and the mapping $U_{\varepsilon}(t, r): \mathcal{E} \rightarrow \mathcal{E}$ is continuous.

The main result of this section can be stated as follows.

Theorem 3.1 Let assumptions (1.2)-(1.4) be satisfied. For any $\varepsilon \in[0,1]$, there exists a pullback attractor $\mathscr{A}_{\varepsilon}=\left\{A_{\varepsilon}(t)\right\}_{t \in \mathbb{R}}$ for Eq. (1.1) such that

$$
\lim _{\varepsilon \rightarrow \varepsilon_{0}} \sup _{t \in[a, b]} \operatorname{dist}_{\mathcal{E}}\left(A_{\varepsilon}(t), A_{\varepsilon_{0}}(t)\right)=0 \quad \text { for all } \varepsilon_{0} \in[0,1] \text { and }[a, b] \subset \mathbb{R}
$$

and

$$
\bigcup_{t \in[a, b]} \bigcup_{\varepsilon \in[0,1]} A_{\varepsilon}(t) \quad \text { is precompact in } \mathcal{E} .
$$

Lemma 3.1 Under assumptions (1.2)-(1.4), we have the estimate

$$
\begin{aligned}
& \left\|\xi_{u}(t)\right\|_{\mathcal{E}}^{2}+e^{-\sigma t} \int_{r}^{t} e^{\sigma s}\left(\|\nabla u(s)\|^{2}+\left\|\partial_{t} u(s)\right\|^{2}\right) d s \\
& \leq C e^{-\sigma(t-r)}\left(\left\|\xi_{u}(r)\right\|_{\mathcal{E}}^{4}+1\right)+C e^{-\sigma t} \int_{r}^{t} e^{\sigma s}\|g(x, s)\|^{2} d s+C
\end{aligned}
$$

for all $\varepsilon \in[0,1], t \geq r$, and any $\xi_{u}(r)=\left(u(r), \partial_{t} u(r)\right) \in \mathcal{E}$, where $\sigma>0$ satisfies (3.16), and $C>0$ is independent of $r, t$, and $\varepsilon$.

Proof Let $F(u)=\int_{0}^{u} f(s) d s$. From (1.3) we obtain that

$$
\begin{aligned}
& \langle f(u), u\rangle \geq-\rho\|u\|^{2}-C_{\rho}, \\
& \langle F(u), 1\rangle \geq-\frac{1}{2} \rho\|u\|^{2}-C_{\rho}, \\
& \langle f(u), u\rangle-\langle F(u), 1\rangle \geq-\frac{1}{2} \rho\|u\|^{2}-C_{\rho}
\end{aligned}
$$

for positive constants $\rho<\lambda_{1}$ and $C_{\rho}$ (see [17] for more detail).

Multiplying Eq. (1.1) by $2\left(\partial_{t} u+\delta u\right)$ and integrating over $\Omega$, we have

$$
\frac{d}{d t} \Lambda(t)+\delta \Lambda(t)+2 \varepsilon\left\|\nabla \partial_{t} u\right\|^{2}+\Gamma(t)=2\left\langle g(x, t), \partial_{t} u+\delta u\right\rangle,
$$


where

$$
\Lambda(t)=(1+\varepsilon \delta)\|\nabla u\|^{2}+\delta\|u\|^{2}+\left\|\partial_{t} u\right\|^{2}+2 \delta\left\langle\partial_{t} u, u\right\rangle+2\langle F(u), 1\rangle+2 C_{\rho}
$$

and

$$
\Gamma(t)=2 \delta\|\nabla u\|^{2}+(2-2 \delta)\left\|\partial_{t} u\right\|^{2}+2 \delta\langle f(u), u\rangle-\delta \Lambda(t) .
$$

Let

$$
0<\delta<\min \left\{\frac{1}{2}, \frac{\lambda_{1}-\rho}{\lambda_{1}+2}\right\} .
$$

By (1.2) and the Sobolev embeddings $H_{0}^{1}(\Omega) \hookrightarrow L^{6}(\Omega) \hookrightarrow L^{4}(\Omega)$ we have

$$
|\langle f(u), u\rangle| \leq C \int_{\Omega}\left(1+|u|^{4}\right) d x \leq C\left(1+\|u\|_{L^{4}}^{4}\right) \leq C\left(1+\|\nabla u\|^{4}\right) .
$$

From (3.4)-(3.9), applying Cauchy's inequality and Poincaré's inequality $\lambda_{1}\|u\|^{2} \leq\|\nabla u\|^{2}$, we deduce that

$$
\begin{aligned}
\Lambda(t) \geq & (1+\varepsilon \delta)\|\nabla u\|^{2}+\delta\|u\|^{2}+\left\|\partial_{t} u\right\|^{2} \\
& -\delta\left\|\partial_{t} u\right\|^{2}-\delta\|u\|^{2}-\rho\|u\|^{2} \\
\geq & \left(1-\rho \lambda_{1}^{-1}\right)\|\nabla u\|^{2}+(1-\delta)\left\|\partial_{t} u\right\|^{2} \\
\geq & C_{1}\left\|\xi_{u}(t)\right\|_{\mathcal{E}}^{2} \\
\Lambda(t) \leq & (1+\varepsilon \delta)\|\nabla u\|^{2}+(2 \delta+\rho)\|u\|^{2}+(1+\delta)\left\|\partial_{t} u\right\|^{2} \\
& +2 C\left(1+\|\nabla u\|^{4}\right)+4 C_{\rho} \\
\leq & (1+2 C)\|\nabla u\|^{4}+\frac{1}{2}\left\|\partial_{t} u\right\|^{4}+\frac{1}{2}(1+\varepsilon \delta)^{2}+\frac{1}{2}\left((2 \delta+\rho) \lambda_{1}^{-1}\right)^{2} \\
& +\frac{1}{2}(1+\delta)^{2}+2 C+4 C_{\rho} \\
\leq & C_{2}\left(\left\|\xi_{u}(t)\right\|_{\mathcal{E}}^{4}+1\right)
\end{aligned}
$$

and

$$
\begin{array}{rl}
\Gamma(t) \geq 2 & 2 \delta\|\nabla u\|^{2}+(2-2 \delta)\left\|\partial_{t} u\right\|^{2}-\delta(1+\varepsilon \delta)\|\nabla u\|^{2} \\
& -\delta^{2}\|u\|^{2}-\delta\left\|\partial_{t} u\right\|^{2}-2 \delta^{2}\left\langle\partial_{t} u, u\right\rangle \\
& +2 \delta(\langle f(u), u\rangle-\langle F(u), 1\rangle)-2 \delta C_{\rho} \\
\geq & \left(2 \delta-\delta(1+\varepsilon \delta)-2 \delta^{2} \lambda_{1}^{-1}-\delta \rho \lambda_{1}^{-1}\right)\|\nabla u\|^{2} \\
& +\left(2-2 \delta-\delta-\delta^{2}\right)\left\|\partial_{t} u\right\|^{2}-4 \delta C_{\rho} \\
\geq & \lambda_{1}^{-1} \delta\left(\left(\lambda_{1}-\rho\right)-\delta\left(\lambda_{1}+2\right)\right)\|\nabla u\|^{2} \\
& +(2-4 \delta)\left\|\partial_{t} u\right\|^{2}-4 \delta C_{\rho}
\end{array}
$$




$$
\geq C_{3}\left\|\xi_{u}(t)\right\|_{\mathcal{E}}^{2}-C_{4}
$$

where $C_{1}=\min \left\{1-\rho \lambda_{1}^{-1}, 1-\delta\right\}, C_{2}=4+\left(2 \lambda_{1}^{-1}+1\right)^{2}+2 C+4 C_{\rho}, C_{3}=\min \left\{\lambda_{1}^{-1} \delta\left(\left(\lambda_{1}-\rho\right)-\right.\right.$ $\left.\left.\delta\left(\lambda_{1}+2\right)\right), 2-4 \delta\right\}$, and $C_{4}=4 \delta C_{\rho}$.

By (3.8) and Cauchy's inequality we observe that

$$
\begin{aligned}
& 2\left\langle g(x, t), \partial_{t} u+\delta u\right\rangle \\
& \quad \leq 2\left\langle g(x, t), \partial_{t} u\right\rangle+2\langle g(x, t), \delta u\rangle \\
& \quad \leq 4 C_{3}^{-1}\|g(x, t)\|^{2}+\frac{C_{3}}{2}\left(\delta^{2}\|u\|^{2}+\left\|\partial_{t} u\right\|^{2}\right) \\
& \quad \leq 4 C_{3}^{-1}\|g(x, t)\|^{2}+\frac{C_{3}}{2}\left\|\xi_{u}(t)\right\|_{\mathcal{E}}^{2} .
\end{aligned}
$$

Combining (3.10)-(3.13), we estimate (3.7) as follows:

$$
\frac{d}{d t} \Lambda(t)+\delta \Lambda(t)+\varepsilon\left\|\nabla \partial_{t} u\right\|^{2} \leq 4 C_{3}^{-1}\|g(x, t)\|^{2}+C_{4}
$$

Multiplying this inequality by $e^{\sigma t}$, we have

$$
\frac{d}{d t}\left(e^{\sigma t} \Lambda(t)\right)+(\delta-\sigma) e^{\sigma t} \Lambda(t) \leq 4 C_{3}^{-1} e^{\sigma t}\|g(x, t)\|^{2}+C_{4} e^{\sigma t}
$$

where

$$
0<2 \sigma<\delta
$$

Integrating (3.15) from $r$ to $t$ and considering (3.10)-(3.11), we have

$$
\begin{aligned}
& C_{1} e^{\sigma t}\left\|\xi_{u}(t)\right\|_{\mathcal{E}}^{2}+C_{1}(\delta-\sigma) \int_{r}^{t} e^{\sigma s}\left\|\xi_{u}(s)\right\|_{\mathcal{E}}^{2} d s \\
& \leq C_{2} e^{\sigma r}\left(\left\|\xi_{u}(r)\right\|_{\mathcal{E}}^{4}+1\right)+4 C_{3}^{-1} \int_{r}^{t} e^{\sigma s}\|g(x, s)\|^{2} d s+C_{4} \sigma^{-1} e^{\sigma t} .
\end{aligned}
$$

After a simple computation, we arrive at

$$
\begin{aligned}
& \left\|\xi_{u}(t)\right\|_{\mathcal{E}}^{2}+e^{-\sigma t} \int_{r}^{t} e^{\sigma s}\left\|\xi_{u}(s)\right\|_{\mathcal{E}}^{2} d s \\
& \leq C_{5}\left(e^{-\sigma(t-r)}\left(\left\|\xi_{u}(r)\right\|_{\mathcal{E}}^{4}+1\right)+e^{-\sigma t} \int_{r}^{t} e^{\sigma s}\|g(x, s)\|^{2} d s+1\right),
\end{aligned}
$$

where $C_{5}=\left(C_{1}(\delta-\sigma)\right)^{-1}\left(C_{2}+4 C_{3}^{-1}+C_{4} \sigma^{-1}\right)$. The proof is completed.

Corollary 3.1 Assume (1.2)-(1.4). Then, for every $\varepsilon \in[0,1]$, the process $U_{\varepsilon}(\cdot, \cdot)$ has a pullback absorbing family $\mathscr{D}=\{D(t)\}_{t \in \mathbb{R}}$ in $\mathcal{E}$, which is independent of $\varepsilon \in[0,1]$ and satisfies (2.2). 
Proof Set

$$
\begin{aligned}
& R(t)=\left(2 C e^{-\sigma t} \int_{-\infty}^{t} e^{\sigma s}\|g(x, s)\|^{2} d s+2 C\right)^{1 / 2}, \\
& D(t)=\left\{\xi \in \mathcal{E} \mid\|\xi\|_{\mathcal{E}} \leq R(t)\right\},
\end{aligned}
$$

where $C$ given by (3.3). By Lemma 3.1 the family $\mathscr{D}=\{D(t)\}_{t \in \mathbb{R}}$ is pullback absorbing for every $U_{\varepsilon}(\cdot, \cdot)$.

Choose $\gamma>0$ satisfying

$$
2 \sigma<\gamma<\delta
$$

where $\delta>0$ is given by (3.8).

From the proof of Lemma 3.1 we easily check that inequality (3.3) still holds if $\sigma$ is replaced by $\gamma$, that is,

$$
\begin{aligned}
\left\|\xi_{u}(t)\right\|_{\mathcal{E}}^{2} & \leq C e^{-\gamma \tau}\left((R(t-\tau))^{4}+1\right)+C e^{-\gamma t} \int_{t-\tau}^{t} e^{(\gamma-\sigma) s} e^{\sigma s}\|g(x, s)\|^{2} d s+C \\
& \leq C e^{-\gamma \tau}\left((R(t-\tau))^{4}+1\right)+C e^{-\sigma t} \int_{-\infty}^{t} e^{\sigma s}\|g(x, s)\|^{2} d s+C
\end{aligned}
$$

for all $u_{t-\tau} \in D(t-\tau)$.

By (3.18) and (3.20) we observe that

$$
e^{-\gamma \tau}(R(t-\tau))^{4} \stackrel{\tau \rightarrow \infty}{\longrightarrow} 0
$$

which, together with (3.19), implies that the family $\{D(t)\}_{t \in \mathbb{R}}$ satisfies (2.2).

Lemma 3.2 Under assumptions (1.2)-(1.4), for any $\left\{t_{n}\right\}_{n \in \mathbb{N}} \subset[a, b]$ such that $t_{n} \stackrel{n \rightarrow \infty}{\longrightarrow} t_{0}$, $\left\{\varepsilon_{n}\right\}_{n \in \mathbb{N}} \subset[0,1]$ such that $\varepsilon_{n} \stackrel{n \rightarrow \infty}{\longrightarrow} \varepsilon_{0}$, and $\left\{x_{n}\right\}_{n \in \mathbb{N}} \subset \mathcal{E}$ such that $x_{n} \stackrel{n \rightarrow \infty}{\longrightarrow} x_{0}$, we have

$$
\left\|U_{\varepsilon_{n}}\left(t_{n}, a-\tau\right) x_{n}-U_{\varepsilon_{0}}\left(t_{0}, a-\tau\right) x_{0}\right\|_{\mathcal{E}^{-1}} \stackrel{n \rightarrow \infty}{\longrightarrow} 0, \quad \forall \tau \geq 0 .
$$

Proof Set $\xi_{u_{n}}(t)=\left(u_{n}(t), \partial_{t} u_{n}(t)\right)=U_{\varepsilon_{n}}(t, a-\tau) x_{n}$ and $\xi_{u_{0}}(t)=\left(u_{0}(t), \partial_{t} u_{0}(t)\right)=U_{\varepsilon_{n}}(t, a-$ $\tau) x_{0}$ be solutions of Eq. (1.1) with initial data $\xi_{u_{n}}(a-\tau)=x_{n}$ and $\xi_{u_{0}}(a-\tau)=x_{0}$, respectively. Set $z_{n}=u_{n}-u_{0}$, which solves the equation

$$
\partial_{t}^{2} z_{n}+\partial_{t} z_{n}-\Delta z_{n}-\varepsilon_{0} \Delta \partial_{t} z_{n}=-\left(f\left(u_{n}\right)-f\left(u_{0}\right)\right)+\left(\varepsilon_{n}-\varepsilon_{0}\right) \Delta \partial_{t} u_{n}
$$

with initial condition $\xi_{z_{n}}(a-\tau)=x_{n}-x_{0}$.

Let us introduce the functional

$$
\Pi(t)=\left(1+\varepsilon_{0} \delta\right)\left\|z_{n}\right\|^{2}+\delta\left\|z_{n}\right\|_{H^{-1}}^{2}+\left\|\partial_{t} z_{n}\right\|_{H^{-1}}^{2}+2 \delta\left\langle(-\Delta)^{-\frac{1}{2}} \partial_{t} z_{n},(-\Delta)^{-\frac{1}{2}} z_{n}\right\rangle .
$$

Taking $0<\delta<1$, we observe that

$$
(1-\delta)\left\|\xi_{z_{n}}(t)\right\|_{\mathcal{E}^{-1}}^{2} \leq \Pi(t) \leq 2\left(1+\lambda_{1}^{-1}\right)\left\|\xi_{z_{n}}(t)\right\|_{\mathcal{E}^{-1}}^{2} .
$$


Multiplying (3.24) by $(-\Delta)^{-1}\left(\partial_{t} z_{n}+\delta z_{n}\right)$ and integrating over $\Omega$, we have

$$
\begin{aligned}
\frac{d}{d t} \Pi(t)+2 \delta\left\|z_{n}\right\|^{2}+(2-2 \delta)\left\|\partial_{t} z_{n}\right\|_{H^{-1}}^{2}+2 \varepsilon_{0}\left\|\partial_{t} z_{n}\right\|^{2} \\
=-2\left\langle f\left(u_{n}\right)-f\left(u_{0}\right),(-\Delta)^{-1}\left(\partial_{t} z_{n}+\delta z_{n}\right)\right\rangle \\
\quad-2\left(\varepsilon_{n}-\varepsilon_{0}\right)\left\langle-\Delta \partial_{t} u_{n},(-\Delta)^{-1}\left(\partial_{t} z_{n}+\delta z_{n}\right)\right\rangle .
\end{aligned}
$$

By (1.2), (3.25), and the embeddings $\mathcal{H}^{1} \hookrightarrow L^{6}(\Omega), \mathcal{H}^{0} \hookrightarrow \mathcal{H}^{-1}$, applying Cauchy's and Hölder's inequalities, the right-hand side of the above equality can be estimated as follows:

$$
\begin{aligned}
& \left.2|| f\left(u_{n}\right)-f\left(u_{0}\right),(-\Delta)^{-1}\left(\partial_{t} z_{n}+\delta z_{n}\right)\right\rangle \mid \\
& \quad \leq C \int_{\Omega}\left(\left|u_{n}\right|^{2}+\left|u_{0}\right|^{2}+1\right)\left|z_{n}\right|\left|(-\Delta)^{-1}\left(\partial_{t} z_{n}+\delta z_{n}\right)\right| d x \\
& \quad \leq C\left(\int_{\Omega}\left(\left|u_{n}\right|^{6}+\left|u_{0}\right|^{6}+1\right) d x\right)^{\frac{1}{3}}\left(\int_{\Omega}\left|z_{n}\right|^{2} d x\right)^{\frac{1}{2}}\left(\int_{\Omega}\left|(-\Delta)^{-1}\left(\partial_{t} z_{n}+\delta z_{n}\right)\right|^{6} d x\right)^{\frac{1}{6}} \\
& \quad \leq C\left(\left\|u_{n}\right\|_{\mathcal{H}^{1}}^{2}+\left\|u_{0}\right\|_{\mathcal{H}^{1}}^{2}+1\right)\left\|z_{n}\right\|\left(\left\|(-\Delta)^{-1} \partial_{t} z_{n}\right\|_{\mathcal{H}^{1}}+\left\|(-\Delta)^{-1} z_{n}\right\|_{\mathcal{H}^{1}}\right) \\
& \quad=C\left(\left\|u_{n}\right\|_{\mathcal{H}^{1}}^{2}+\left\|u_{0}\right\|_{\mathcal{H}^{1}}^{2}+1\right)\left\|z_{n}\right\|\left(\left\|\partial_{t} z_{n}\right\|_{\mathcal{H}^{-1}}+\left\|z_{n}\right\|_{\mathcal{H}^{-1}}\right) \\
& \quad \leq C\left(\left\|u_{n}\right\|_{\mathcal{H}^{1}}^{2}+\left\|u_{0}\right\|_{\mathcal{H}^{1}}^{2}+1\right) \Pi(t)
\end{aligned}
$$

and

$$
\begin{aligned}
& 2\left(\varepsilon_{n}-\varepsilon_{0}\right)\left|\left\langle-\Delta \partial_{t} u_{n},(-\Delta)^{-1}\left(\partial_{t} z_{n}+\delta z_{n}\right)\right\rangle\right| \\
& \quad \leq 2\left(\varepsilon_{n}-\varepsilon_{0}\right)\left|\left\langle(-\Delta)^{\frac{1}{2}} \partial_{t} u_{n},(-\Delta)^{-\frac{1}{2}}\left(\partial_{t} z_{n}+\delta z_{n}\right)\right\rangle\right| \\
& \quad \leq 2\left(\varepsilon_{n}-\varepsilon_{0}\right)^{2}\left\|\partial_{t} u_{n}\right\|_{\mathcal{H}^{1}}^{2}+\left\|\partial_{t} z_{n}\right\|_{\mathcal{H}^{-1}}^{2}+\delta^{2}\left\|z_{n}\right\|_{\mathcal{H}^{-1}}^{2} .
\end{aligned}
$$

Hence

$$
\frac{d}{d t} \Pi(t) \leq C\left(\left\|u_{n}\right\|_{\mathcal{H}^{1}}^{2}+\left\|u_{0}\right\|_{\mathcal{H}^{1}}^{2}+1\right) \Pi(t)+2 \varepsilon_{n}^{-1}\left(\varepsilon_{n}-\varepsilon_{0}\right)^{2} \cdot \varepsilon_{n}\left\|\partial_{t} u_{n}\right\|_{\mathcal{H}^{1}}^{2} .
$$

Integrating (3.14) from $a-\tau$ to $b$, we get

$$
\int_{a-\tau}^{b}\left(\|u(t)\|_{\mathcal{H}^{1}}^{2}+\varepsilon\left\|\partial_{t} u(t)\right\|_{\mathcal{H}^{1}}^{2}\right) d t \leq Q \quad \text { for all } \varepsilon \in[0,1]
$$

where $Q>0$ depends on $a-\tau, b$, and $\left\|\xi_{u}(a-\tau)\right\|_{\mathcal{E}}$ but is independent of $\varepsilon$.

By (3.25) and (3.27) an application of Gronwall's inequality to (3.26) entails

$$
\sup _{t \in[a, b]}\left\|\xi_{z_{n}}(t)\right\|_{\mathcal{E}^{-1}}^{2} \leq Q\left\|\xi_{z_{n}}(a-\tau)\right\|_{\mathcal{E}^{-1}}^{2}+Q \varepsilon_{n}^{-1}\left(\varepsilon_{n}-\varepsilon_{0}\right)^{2},
$$

where $Q>0$ depends on $a-\tau, b$, and $D(a-\tau)$ but is independent of $\varepsilon$.

Taking the limits as $n \rightarrow \infty$ yields

$$
\sup _{t \in[a, b]}\left\|U_{\varepsilon_{n}}(t, a-\tau) x_{n}-U_{\varepsilon_{0}}(t, a-\tau) x_{0}\right\|_{\mathcal{E}^{-1}} \stackrel{n \rightarrow \infty}{\longrightarrow} 0 .
$$


Since $U_{\mathcal{E}}(\cdot, a-\tau) x \in C([a-\tau, b], \mathcal{E})$, we have

$$
\begin{aligned}
& \left\|U_{\varepsilon_{n}}\left(t_{n}, a-\tau\right) x_{n}-U_{\varepsilon_{0}}\left(t_{0}, a-\tau\right) x_{0}\right\|_{\mathcal{E}^{-1}} \\
& \quad \leq\left\|U_{\varepsilon_{n}}\left(t_{n}, a-\tau\right) x_{n}-U_{\varepsilon_{0}}\left(t_{n}, a-\tau\right) x_{0}\right\|_{\mathcal{E}^{-1}} \\
& \quad+\left\|U_{\varepsilon_{0}}\left(t_{n}, a-\tau\right) x_{0}-U_{\varepsilon_{0}}\left(t_{0}, a-\tau\right) x_{0}\right\|_{\mathcal{E}^{-1}} \stackrel{n \rightarrow \infty}{\longrightarrow} 0 .
\end{aligned}
$$

This finishes the proof.

To obtain the regularity estimates, we will apply the ideas of Zelik [25].

Split the solution $U(t, r) \xi_{u}(r)=u(t)$ of Eq. (1.1) as follows:

$$
U(t, r) \xi_{u}(r)=V(t, r) \xi_{v}(r)+W(t, r) \xi_{w}(r)
$$

where $V(t, r) \xi_{v}(r)=\xi_{v}(t)$ and $W(t, r) \xi_{w}(r)=\xi_{w}(t)$ solve the following equations, respectively:

$$
\left\{\begin{array}{l}
\partial_{t}^{2} v+\partial_{t} v-\varepsilon \Delta \partial_{t} v-\Delta v+f(v)+L v=0, \\
\xi_{v}(r)=\xi_{u}(r),\left.\quad v(x, t)\right|_{\partial \Omega \times[r, \infty)}=0,
\end{array}\right.
$$

and

$$
\left\{\begin{array}{l}
\partial_{t}^{2} w+\partial_{t} w-\varepsilon \Delta \partial_{t} w-\Delta w+f(u)-f(v)=L v(t)+g(x, t), \\
\xi_{w}(r)=0,\left.\quad w(x, t)\right|_{\partial \Omega \times[r, \infty)}=0,
\end{array}\right.
$$

where $L>0$ will be settled in the proof of Lemma 3.3.

Lemma 3.3 Assume (1.2)-(1.4). Then for any $[a, b] \subset \mathbb{R}$ and $\mu>0$, there exists $T_{\mu}>0$ such that the following estimate holds:

$$
\sup _{\substack{\xi_{v}(a-\tau) \in D(a-\tau) \\ t \in[a, b] \\ \varepsilon \in[0,1]}}\left\|V(t, a-\tau) \xi_{\nu}(a-\tau)\right\|_{\mathcal{E}}^{2} \leq \mu \quad \text { for all } \tau \geq T_{\mu}
$$

where $\{D(t)\}_{t \in \mathbb{R}}$ is defined by (3.19).

Proof Let $\gamma>0$ satisfy (3.20). Using the same argument as in the proof of Lemma 3.1, we have

$$
\sup _{\substack{\xi_{v}(a-\tau) \in D(a-\tau) \\ \varepsilon \in[0,1]}}\left\|\xi_{v}(t)\right\|_{\mathcal{E}}^{2} \leq C e^{-\gamma(t-a+\tau)}\left((R(a-\tau))^{4}+1\right)+C .
$$

Then we can find $T>0$ large enough such that

$$
\sup _{\substack{\xi_{v}(a-\tau) \in D(a-\tau) \\ t \in[a, b] \\ \varepsilon \in[0,1]}}\left\|\xi_{v}(t)\right\|_{\mathcal{E}}^{2} \leq C \quad \text { for all } \tau \geq T
$$


From (1.3), noting that $f(0)=0$, we have

$$
\langle f(v), v\rangle \geq-K\|v\|^{2} \quad \text { and } \quad\langle F(u), 1\rangle \geq-\frac{K}{2}\|v\|^{2}
$$

for some positive $K$.

Let $0<\delta<1$ and $L>K$. Multiplying (3.31) by $\partial_{t} v+\delta v$ and integrating over $\Omega$, we have

$$
\frac{d}{d t} \Phi_{\varepsilon}(t)+\beta\left\|\xi_{v}(t)\right\|_{\mathcal{E}}^{2} \leq 0 \quad \text { for all } \varepsilon \in[0,1]
$$

where $\beta=\min \{2(1-\delta), 2 \delta\}$, and

$$
\begin{aligned}
\Phi_{\varepsilon}(t) & :=\Phi_{\varepsilon}\left(V(t, a-\tau) \xi_{v}(a-\tau)\right) \\
& :=\left\|\partial_{t} v\right\|^{2}+\|\nabla v\|^{2}+\delta\|v\|^{2}+\varepsilon \delta\|\nabla v\|^{2}+2 \delta\left\langle\partial_{t} v, v\right\rangle+2\langle F(v), 1\rangle+L\|v\|^{2} .
\end{aligned}
$$

In light of (1.2) and (3.35), noting that $L>K$, we easily realize that $\Phi_{\varepsilon}(t)$ fulfills the inequalities

$$
C_{1}\left\|\xi_{v}(t)\right\|_{\mathcal{E}}^{2} \leq \Phi_{\varepsilon}(t) \leq C_{2}\left(\left\|\xi_{v}(t)\right\|_{\mathcal{E}}^{4}+1\right)
$$

for some suitable positive constants $C_{1}, C_{2}$, which are independent of $\varepsilon$.

By (3.34) and (3.38) there exists $T>0$ such that

$$
\sup _{\substack{\xi_{v}(a-\tau) \in D(a-\tau) \\ t \in[a, b] \\ \varepsilon \in[0,1]}} \Phi_{\varepsilon}\left(V(t, a-\tau) \xi_{v}(a-\tau)\right)<\widetilde{C} \quad \text { for all } \tau \geq T
$$

where $\widetilde{C}>0$ is independent of $a-\tau, t$, and $\varepsilon$.

Next, we claim that for any $\eta>0$, there exist $\tau_{\eta}>a$ and $t_{\eta} \in\left[a-\tau_{\eta}, a\right]$ such that

$$
\left.\frac{d}{d t} \Phi_{\varepsilon}\left(V\left(t, a-\tau_{\eta}\right) \xi_{v}\left(a-\tau_{\eta}\right)\right)\right|_{t=t_{\eta}} \geq-\eta
$$

for all $\varepsilon \in[0,1]$ and $\xi_{v}\left(a-\tau_{\eta}\right) \in D\left(a-\tau_{\eta}\right)$. If not, then for $\tau_{n} \stackrel{n \rightarrow \infty}{\longrightarrow} \infty$, there exist $\varepsilon_{n} \in[0,1]$ and $\xi_{v}\left(a-\tau_{n}\right) \in D\left(a-\tau_{n}\right)$ such that

$$
\frac{d}{d t} \Phi_{\varepsilon_{n}}\left(V\left(t, a-\tau_{n}\right) \xi_{v}\left(a-\tau_{n}\right)\right)<-\eta \quad \text { for all } t \in\left[a-\tau_{n}, a\right] \text { and } n \in \mathbb{N}
$$

Hence, integrating the above inequality over $\left[a-\frac{\widetilde{C}}{\eta}, a\right]$ and considering (3.39), we get that there exists $N \in \mathbb{N}$ large enough such that

$$
\begin{aligned}
& \Phi_{\varepsilon_{n}}\left(V\left(a, a-\tau_{n}\right) \xi_{v}\left(a-\tau_{n}\right)\right) \\
& \quad<-\widetilde{C}+\Phi_{\varepsilon_{n}}\left(V\left(a-\frac{\widetilde{C}}{\eta}, a-\tau_{n}\right) \xi_{v}\left(a-\tau_{n}\right)\right)<0 \quad \text { for all } n \geq N
\end{aligned}
$$

which contradicts the positivity of $\Phi_{\varepsilon}(t)$ (see (3.38)). Thus (3.40) is correct. 
Exploiting (3.36) and (3.40), we have

$$
\sup _{\substack{\xi_{v}\left(a-\tau_{\eta}\right) \in D\left(a-\tau_{\eta}\right) \\ \varepsilon \in[0,1]}} \beta\left\|\xi_{v}\left(t_{\eta}\right)\right\|_{\mathcal{E}}^{2} \leq \eta,
$$

which implies

$$
\begin{aligned}
& \sup _{\substack{\xi_{\nu}\left(a-\tau_{\eta}\right) \in D\left(a-\tau_{\eta}\right) \\
\varepsilon \in[0,1]}} \Phi_{\varepsilon}\left(V\left(t_{\eta}, a-\tau_{\eta}\right) \xi_{\nu}\left(a-\tau_{\eta}\right)\right) \\
& \leq \sup _{\substack{\xi_{\nu}\left(a-\tau_{\eta}\right) \in D\left(a-\tau_{\eta}\right) \\
\varepsilon \in[0,1]}}\left\{\Phi_{\varepsilon}\left(V\left(t_{\eta}, a-\tau_{\eta}\right) \xi_{v}\left(a-\tau_{\eta}\right)\right) \mid \beta\left\|\xi_{v}\left(t_{\eta}\right)\right\|_{\mathcal{E}}^{2} \leq \eta\right\} .
\end{aligned}
$$

Inequality (3.36) implies that $\Phi_{\varepsilon}(t)$ is a nonincreasing function. Note that $t_{\eta} \in\left[a-\tau_{\eta}, a\right]$ and hence

$$
\Phi_{\varepsilon}\left(V\left(t, a-\tau_{\eta}\right) \xi_{v}\left(a-\tau_{\eta}\right)\right) \leq \Phi_{\varepsilon}\left(V\left(t_{\eta}, a-\tau_{\eta}\right) \xi_{v}\left(a-\tau_{\eta}\right)\right) \quad \text { for all } t \geq a
$$

which, together with (3.44), yields

$$
\begin{aligned}
& \sup _{\substack{\xi_{\nu}\left(a-\tau_{\eta}\right) \in D\left(a-\tau_{\eta}\right) \\
\varepsilon \in[0,1] \\
t \in[a, b]}} \Phi_{\varepsilon}\left(V\left(t, a-\tau_{\eta}\right) \xi_{\nu}\left(a-\tau_{\eta}\right)\right) \\
& \leq \sup _{\substack{\xi_{\nu}\left(a-\tau_{\eta}\right) \in D\left(a-\tau_{\eta}\right) \\
\varepsilon \in[0,1]}}\left\{\Phi_{\varepsilon}\left(V\left(t_{\eta}, a-\tau_{\eta}\right) \xi_{v}\left(a-\tau_{\eta}\right)\right) \mid \beta\left\|\xi_{v}\left(t_{\eta}\right)\right\|_{\mathcal{E}}^{2} \leq \eta\right\} .
\end{aligned}
$$

Since $V(\cdot, \cdot)$ forms a process on $\mathcal{E}$, by (3.34) we can find $T_{\eta}>0$ large enough, which depends on $\tau_{\eta}$, such that

$$
V\left(a-\tau_{\eta}, a-\tau\right) D(a-\tau) \subset D\left(a-\tau_{\eta}\right) \quad \text { for all } \tau \geq T_{\eta} \text { and all } \varepsilon \in[0,1]
$$

Thus, in view of (3.46), we arrive at

$$
\begin{aligned}
& \sup _{\substack{\xi_{\nu}(a-\tau) \in D(a-\tau) \\
\varepsilon \in[0,1] \\
t \in[a, b]}} \Phi_{\varepsilon}\left(V(t, a-\tau) \xi_{\nu}(a-\tau)\right) \\
& \leq \sup _{\substack{\xi_{\nu}\left(a-\tau_{\eta}\right) \in D\left(a-\tau_{\eta}\right) \\
\varepsilon \in[0,1]}}\left\{\Phi_{\varepsilon}\left(V\left(t_{\eta}, a-\tau_{\eta}\right) \xi_{\nu}\left(a-\tau_{\eta}\right)\right) \mid \beta\left\|\xi_{\nu}\left(t_{\eta}\right)\right\|_{\mathcal{E}}^{2} \leq \eta\right\}
\end{aligned}
$$

for all $\tau \geq T_{\eta}$.

From the definition of $\Phi_{\varepsilon}(t)$ we easily to check that $\Phi_{\varepsilon}\left(\left(v_{1}, v_{2}\right)\right) \rightarrow 0$ as $\left\|\left(v_{1}, v_{2}\right)\right\|_{\mathcal{E}}^{2} \rightarrow 0$ uniformly with respect to $\varepsilon \in[0,1]$. Then from the above analysis, fixing $\mu>0$ small enough, we can choose $\eta>0, \tau_{\eta}>0$, and $t_{\eta} \in\left[a-\tau_{\eta}, a\right]$ such that

$$
\sup _{\substack{\xi_{v}\left(a-\tau_{\eta}\right) \in D\left(a-\tau_{\eta}\right) \\ \varepsilon \in[0,1]}} \beta\left\|\xi_{v}\left(t_{\eta}\right)\right\|_{\mathcal{E}}^{2} \leq \eta
$$




$$
\Longrightarrow \quad \sup _{\substack{\xi_{\nu}\left(a-\tau_{\eta}\right) \in D\left(a-\tau_{\eta}\right) \\ \varepsilon \in[0,1]}} \Phi_{\varepsilon}\left(V\left(t_{\eta}, a-\tau_{\eta}\right) \xi_{v}\left(a-\tau_{\eta}\right)\right) \leq C_{1} \mu
$$

which, together with (3.38) and (3.47), implies

$$
\sup _{\substack{\xi_{v}(a-\tau) \in D(a-\tau) \\ t \in[a, b] \\ \varepsilon \in[0,1]}}\left\|\xi_{v}(t)\right\|_{\mathcal{E}}^{2} \leq \mu \quad \text { for all } \tau \geq T
$$

where $T>0$ large enough. This completes the proof.

To obtain the regularity of $w$, we need the following result in [25].

Lemma 3.4 Let $\alpha \in\left[0, \frac{1}{2}\right)$. Then

$$
\begin{aligned}
& \left\|u_{1} \cdot(-\Delta)^{\alpha-1} u_{2}\right\|_{L^{3}} \leq C\left\|u_{1}\right\|_{\mathcal{H}^{\alpha+1}}\left\|u_{2}\right\|_{\mathcal{H}^{\alpha-1}}, \\
& \left\|u_{3} \cdot(-\Delta)^{\alpha-1} u_{2}\right\|_{L^{\frac{3}{2}}} \leq C\left\|u_{3}\right\|_{\mathcal{H}^{\alpha}}\left\|u_{2}\right\|_{\mathcal{H}^{\alpha-1}}
\end{aligned}
$$

for all $u_{1} \in \mathcal{H}^{\alpha+1}, u_{2} \in \mathcal{H}^{\alpha-1}$, and $u_{3} \in \mathcal{H}^{\alpha}$, where the positive constant $C$ depends on $\alpha$ but is independent of $u_{1}$ and $u_{2}$.

Lemma 3.5 Under the same assumptions of Lemma 3.3, for any $\alpha \in\left[0, \frac{1}{2}\right),[a, b] \subset \mathbb{R}$, and $M>0$, we have

$$
\sup _{\substack{\left\|\xi_{w}(a-\tau)\right\| \leq M \\ t \in[a, b] \\ \varepsilon \in[0,1]}}\left\|W(t, a-\tau) \xi_{w}(a-\tau)\right\|_{\mathcal{E}^{\alpha}} \leq Q
$$

where $Q>0$ depends on $\alpha, a-\tau, b$, and $M$ but is independent of $\varepsilon$.

Proof Multiplying Eq. (3.32) by $(-\Delta)^{\alpha} \partial_{t} w$ and integrating over $\Omega$, we have

$$
\begin{aligned}
& \frac{d}{d t}\left(\left\|\partial_{t} w\right\|_{\mathcal{H}^{\alpha}}^{2}+\|w\|_{\mathcal{H}^{\alpha+1}}^{2}\right)+2\left\|\partial_{t} w\right\|_{\mathcal{H}^{\alpha}}^{2}+2 \varepsilon\left\|\partial_{t} w\right\|_{\mathcal{H}^{\alpha+1}}^{2} \\
& \quad \leq 2\left|\left\langle f(v+w)-f(v),(-\Delta)^{\alpha} \partial_{t} w\right\rangle\right|+2\left|\left\langle L v+g(x, t),(-\Delta)^{\alpha} \partial_{t} w\right\rangle\right| .
\end{aligned}
$$

Applying (1.2) and the embeddings $\mathcal{H}^{1} \hookrightarrow L^{6}(\Omega), \mathcal{H}^{\alpha+1} \hookrightarrow \mathcal{H}^{2 \alpha}$, we deduce that

$$
\begin{aligned}
& 2\left|\left\langle f(v+w)-f(v),(-\Delta)^{\alpha} \partial_{t} w\right\rangle\right| \\
& \quad \leq C \int_{\Omega}\left(1+|u|^{2}+|v|^{2}\right)(|u|+|v|)\left|(-\Delta)^{\alpha} \partial_{t} w\right| d x \\
& \quad \leq C\left(\int_{\Omega}\left(1+|u|^{6}+|v|^{6}\right) d x\right)^{\frac{1}{3}}\left(\int_{\Omega}\left(|u|^{6}+|v|^{6}\right) d x\right)^{\frac{1}{6}}\left(\int_{\Omega}\left|(-\Delta)^{\alpha} \partial_{t} w\right|^{2} d x\right)^{\frac{1}{2}} \\
& \quad \leq C\left(1+\|u\|_{\mathcal{H}^{1}}^{2}+\|v\|_{\mathcal{H}^{1}}^{2}\right)\left(\|u\|_{\mathcal{H}^{1}}+\|v\|_{\mathcal{H}^{1}}\right)\left\|\partial_{t} w\right\|_{\mathcal{H}^{2 \alpha}} \\
& \quad \leq \frac{C}{\varepsilon}\left(1+\|u\|_{\mathcal{H}^{1}}^{6}+\|v\|_{\mathcal{H}^{1}}^{6}\right)+\frac{\varepsilon}{2}\left\|\partial_{t} w\right\|_{\mathcal{H}^{\alpha+1}}^{2}
\end{aligned}
$$


and

$$
2\left|\left\langle L v+g(x, t),(-\Delta)^{\alpha} \partial_{t} w\right\rangle\right| \leq \frac{C}{\varepsilon}\left(\|v\|^{2}+\|g(x, t)\|^{2}\right)+\frac{\varepsilon}{2}\left\|\partial_{t} w\right\|_{\mathcal{H}^{\alpha+1}}^{2} .
$$

As a consequence, inequality (3.52) improves to

$$
\frac{d}{d t}\left(\left\|\partial_{t} w\right\|_{\mathcal{H}^{\alpha}}^{2}+\|w\|_{\mathcal{H}^{\alpha+1}}^{2}\right)+\varepsilon\left\|\partial_{t} w\right\|_{\mathcal{H}^{\alpha+1}}^{2} \leq \frac{C}{\varepsilon}\left(1+\|u\|_{\mathcal{H}^{1}}^{6}+\|v\|_{\mathcal{H}^{1}}^{6}+\|g(x, t)\|^{2}\right)
$$

Integrating this inequality from $a-\tau$ to $t$ and considering Lemmas 3.1 and 3.3, we deduce

$$
\int_{a-\tau}^{t} \varepsilon^{2}\left\|\partial_{t} w(s)\right\|_{\mathcal{H}^{\alpha+1}}^{2} d s \leq Q \quad \text { for all } t \in[a, b]
$$

where the positive constant $Q$ depends on $a-\tau, b$, and $M$ but is independent of $\varepsilon$.

Differentiating Eq. (3.32) with respect to $t$ and setting $\theta(t)=\partial_{t} w$, we have

$$
\partial_{t}^{2} \theta+\partial_{t} \theta-\varepsilon \Delta \partial_{t} \theta-\Delta \theta=-\left(f^{\prime}(u)-f^{\prime}(v)\right) \partial_{t} u-f^{\prime}(v) \theta+L \partial_{t} v(t)+\partial_{t} g(x, t)
$$

with initial condition

$$
\theta(r)=0, \partial_{t} \theta(r)=L u(r)+g(x, r) .
$$

Taking the scalar product of (3.54) with $(-\Delta)^{\alpha-1}\left(\partial_{t} \theta+\delta \theta\right)$ (where $\delta>0$ is small enough), we find that

$$
\begin{aligned}
& \frac{d}{d t} \Psi(t)+\delta \Psi(t) \\
& \leq-\left\langle\left(f^{\prime}(v+w)-f^{\prime}(v)\right) \partial_{t} u,(-\Delta)^{\alpha-1}\left(\partial_{t} \theta+\delta \theta\right)\right\rangle \\
&-\left\langle\left(f^{\prime}(v)-f^{\prime}(0)\right) \theta,(-\Delta)^{\alpha-1}\left(\partial_{t} \theta+\delta \theta\right)\right\rangle \\
&+\left\langle L \partial_{t} v(t)+\partial_{t} g(x, t)-f^{\prime}(0) \theta,(-\Delta)^{\alpha-1}\left(\partial_{t} \theta+\delta \theta\right)\right\rangle \\
&:= I_{1}+I_{2}+I_{3},
\end{aligned}
$$

where

$$
\Psi(t)=\left\|\xi_{\theta}\right\|_{\mathcal{E}^{\alpha-1}}^{2}+\delta\|\theta\|_{\mathcal{H}^{\alpha-1}}^{2}+\varepsilon \delta\|\theta\|_{\mathcal{H}^{\alpha}}^{2}+2 \delta\left\langle\partial_{t} \theta,(-\Delta)^{\alpha-1} \theta\right\rangle
$$

and we easily check that

$$
C_{1}\left\|\xi_{\theta}\right\|_{\mathcal{E}^{\alpha-1}}^{2} \leq \Psi(t) \leq C_{2}\left\|\xi_{\theta}\right\|_{\mathcal{E}^{\alpha-1}}^{2}
$$

for some positive constants $C_{1}$ and $C_{2}$.

Next, we estimate $I_{1}, I_{2}$, and $I_{3}$ one by one.

From (1.2) we have

$$
\left|f^{\prime}(v+w)-f^{\prime}(w)\right| \leq C(1+|v+w|+|v|)|w|,
$$

where $C>0$ is independent of $v, w$, and $\varepsilon$. 
Taking the $\mathcal{H}^{\alpha-1}$-norm of the both sides of Eq. (3.32), applying (1.2), Lemmas 3.1 and 3.3, and the embedding $\mathcal{H}^{1} \hookrightarrow L^{6}(\Omega)$, we derive that

$$
\begin{aligned}
\left\|\varepsilon \partial_{t} w+w\right\|_{\mathcal{H}^{\alpha+1}} & \leq\left\|\partial_{t}^{2} w\right\|_{\mathcal{H}^{\alpha-1}}+C\left(\left\|\partial_{t} w\right\|+\|f(u)\|+\|f(v)\|+\|g(x, t)\|+L\|v\|\right) \\
& \leq\left\|\partial_{t} \theta\right\|_{\mathcal{H}^{\alpha-1}}+Q
\end{aligned}
$$

where $Q>0$ depends on $a-\tau, b$, and $M$ but is independent of $\varepsilon$.

Due to Lemma 3.4 and (3.59) and (3.60), we estimate $I_{i}$ as follows:

$$
\begin{aligned}
I_{1} & \leq C\left(1+\|u\|_{L^{6}}+\|v\|_{L^{6}}\right)\left\|\partial_{t} u\right\|\left\|w \cdot(-\Delta)^{\alpha-1}\left(\partial_{t} \theta+\delta \theta\right)\right\|_{L^{3}} \\
& \leq Q\|w\|_{\mathcal{H}^{\alpha+1}}\left\|\partial_{t} \theta+\delta \theta\right\|_{\mathcal{H}^{\alpha-1}} \\
& \leq Q\left(\left\|w+\varepsilon \partial_{t} w\right\|_{\mathcal{H}^{\alpha+1}}+\varepsilon\left\|\partial_{t} w\right\|_{\mathcal{H}^{\alpha+1}}\right)\left\|\partial_{t} \theta+\delta \theta\right\|_{\mathcal{H}^{\alpha-1}} \\
& \leq Q\left\|\xi_{\theta}\right\|_{\mathcal{E}^{\alpha-1}}^{2}+\varepsilon^{2}\left\|\partial_{t} w\right\|_{\mathcal{H}^{\alpha+1}}^{2}+Q \\
I_{2} & \leq\left\|f^{\prime}(v)-f^{\prime}(0)\right\|_{L^{3}}\left\|\theta \cdot(-\Delta)^{\alpha-1}\left(\partial_{t} \theta+\delta \theta\right)\right\|_{L^{\frac{3}{2}}} \\
& \leq C\left(\|v\|_{\mathcal{H}^{1}}^{2}+1\right)\|\theta\|_{\mathcal{H}^{\alpha}}\left\|\partial_{t} \theta+\delta \theta\right\|_{\mathcal{H}^{\alpha-1}} \\
& \leq Q\left\|\xi_{\theta}\right\|_{\mathcal{E}^{\alpha-1}}^{2},
\end{aligned}
$$

and

$$
I_{3} \leq C\left(\left\|\partial_{t} u\right\|+\left\|\partial_{t} v\right\|+\left\|\partial_{t} g(x, t)\right\|\right)\left\|\partial_{t} \theta+\delta \theta\right\|_{\mathcal{H}^{\alpha-1}} \leq\left\|\xi_{\theta}\right\|_{\mathcal{E}^{\alpha-1}}^{2}+Q,
$$

where $Q>0$ depends on $\alpha, a-\tau, b$, and $M$ but is independent of $\varepsilon$.

Combining (3.58) and (3.61)-(3.63), we simplify (3.56) as follows:

$$
\frac{d}{d t} \Psi(t) \leq Q \Psi(t)+\varepsilon^{2}\left\|\partial_{t} w(t)\right\|_{\mathcal{H}^{\alpha+1}}^{2}+Q .
$$

Applying the Gronwall inequality to (3.64) and using of (3.53), it follows that

$$
\left\|\partial_{t} w(t)\right\|_{\mathcal{H}^{\alpha}}+\left\|\partial_{t}^{2} w\right\|_{\mathcal{H}^{\alpha-1}} \leq Q \quad \text { for all } t \in[a-\tau, b] \text { and } \varepsilon \in[0,1]
$$

provided that $\left\|\xi_{u}(r)\right\| \leq M$.

Now we rewrite Eq. (3.32) as

$$
\varepsilon \partial_{t} w(t)+w(t)=(-\Delta)^{-1} H(t),
$$

where $H(t)=-\partial_{t}^{2} w-\partial_{t} w-(f(u)-f(v))+L v+g(x, t)$ and $(-\Delta)^{-1} H(t) \in C_{b}\left([a-\tau, b], L^{2}(\Omega)\right)$. Applying the variation of constants method and considering the fact that $\xi_{w}(a-\tau)=0$, we obtain

$$
w(t)=\frac{1}{\varepsilon} e^{-\frac{1}{\varepsilon} t} \int_{a-\tau}^{t} e^{\frac{1}{\varepsilon} s}(-\Delta)^{-1} H(s) d s .
$$

From Lemmas 3.1 and 3.3 and (3.65) we find

$$
\|H(t)\|_{\mathcal{H}^{\alpha-1}} \leq Q \quad \text { for all } t \in[a-\tau, b] \text { and } \varepsilon \in[0,1]
$$

where $Q>0$ depends on $\alpha, a-\tau, b$, and $M$ but is independent of $\varepsilon$. 
Finally, combining (3.65) and (3.68) and taking the $\mathcal{H}^{\alpha+1}$-norm of both sides of (3.67), we get the desired conclusion. The proof is finished.

The proof of Theorem 3.1 The existence of pullback attractors follows directly from Theorem 2.1, Corollary 3.1, and Lemmas 3.3 and 3.5. Then by Lemmas 2.1, 3.2, 3.3, and 3.5 we readily check that all assumptions of Theorem 2.2 (with $X=\mathcal{E}$ and $Y=\mathcal{E}^{-1}$ ) are satisfied.

\author{
Acknowledgements \\ We would like to thank the referee for invaluable comments and insightful suggestions. \\ Funding \\ The first author was supported by the Fundamental Research Funds for the Central Universities (No. INS1909). The third \\ author was supported by the National Natural Science Foundation of China (No. 11671075) and the Fundamental \\ Research Funds for the Central Universities (No. 2232021G-13).
}

\title{
Availability of data and materials
}

Not applicable.

\section{Competing interests}

The authors declare that they have no competing interests.

\section{Authors' contributions}

Equal contributions by all authors. All authors read and approved the final manuscript.

\section{Publisher's Note}

Springer Nature remains neutral with regard to jurisdictional claims in published maps and institutional affiliations.

Received: 17 February 2021 Accepted: 2 June 2021 Published online: 10 June 2021

\section{References}

1. Caraballo, T., Carvalho, A.N., Langa, J.A., Rivero, F.: Existence of pullback attractors for pullback asymptotically compact processes. Nonlinear Anal. 72, 1967-1976 (2010)

2. Caraballo, T., Langa, J.A., Robinson, J.C.: Upper semicontinuity of attractors for small random perturbations of dynamical systems. Commun. Partial Differ. Equ. 23, 1557-1581 (1998)

3. Caraballo, T., Łukaszewicz, G., Real, J.: Pullback attractors for asymptotically compact non-autonomous dynamical systems. Nonlinear Anal. 64, 484-498 (2006)

4. Carvalho, A.N., Cholewa, J.W.: Local well posedness for strongly damped wave equations with critical nonlinearities Bull. Aust. Math. Soc. 66, 443-463 (2002)

5. Carvalho, A.N., Cholewa, J.W.: Attractors for strongly damped wave equations with critical nonlinearities. Pac. J. Math. 207, 287-310 (2002)

6. Carvalho, A.N., Langa, J.A., Robinson, J.C.: On the continuity of pullback attractors for evolution processes. Nonlinear Anal. 71, 1812-1824 (2009)

7. Carvalho, A.N., Langa, J.A., Robinson, J.C.: Attractor for Infinite-Dimensional Nonautonomous Dynamical Systems. Springer, Berlin (2013)

8. Cooper, S., Savostianov, A.: Homogenisation with error estimates of attractors for damped semi-linear anisotropic wave equations. Adv. Nonlinear Anal. 9, 745-787 (2020)

9. Dell'Oro, F., Pata, V.: Strongly damped wave equations with critical nonlinearities. Nonlinear Anal. 75, 5723-5735 (2012)

10. Freitas, M.M., Costa, A.L., Araújo, G.M.: Pullback dynamics of a non-autonomous mixture problem in one dimensional solids with nonlinear damping. Commun. Pure Appl. Anal. 19, 785-809 (2020)

11. Ghidagla, J.M., Marzocchi, A.: Longtime behaviour of strongly damped wave equations, global attractors and their dimension. SIAM J. Math. Anal. 22, 879-895 (1991)

12. Ghisi, M., Gobbino, M., Haraux, A.: Quantization of energy and weakly turbulent profiles of solutions to some damped second-order evolution equations. Adv. Nonlinear Anal. 8, 902-927 (2019)

13. Li, Y.R., Gu, A.H., Li, J.: Existence and continuity of bi-spatial random attractors and application to stochastic semilinear Laplacian equations. J. Differ. Equ. 258, 504-534 (2015)

14. Liu, L.F., Fu, X.L.: Existence and upper semicontinuity of pullback attractors of a $p$-Laplacian equation with delay. J. Math. Phys. 58, $082702(2017)$

15. Pata, V., Squassina, M.: On the strongly damped wave equation. Commun. Math. Phys. 253, 511-533 (2005)

16. Pata, V., Zelik, S.: A remark on the damped wave equation. Commun. Pure Appl. Anal. 5, 611-616 (2006)

17. Pata, V., Zelik, S.: Smooth attractors for strongly damped wave equations. Nonlinearity 19, 1495-1506 (2006)

18. Sell, G.R., You, Y.C.: Dynamics of Evolutionary Equations. Applied Mathematical Sciences, vol. 143. Springer, New York (2002)

19. Sun, W.L.: The boundedness and upper semicontinuity of the pullback attractors for a $2 \mathrm{D}$ micropolar fluid flows with delay. Electron. Res. Arch. 28, 1343-1356 (2020) 
20. Wang, B.X.: Asymptotic behavior of stochastic wave equations with critical exponents on $\mathbb{R}^{3}$. Trans. Am. Math. Soc. 363, 3639-3663 (2011)

21. Wang, B.X: Random attractors for non-autonomous stochastic wave equations with multiplicative noise. Discrete Contin. Dyn. Syst., Ser. A 34, 269-300 (2014)

22. Wang, Z.J., Zhou, S.F.: Random attractor for non-autonomous stochastic strongly damped wave equation on unbounded domains. J. Appl. Anal. Comput. 5, 363-387 (2015)

23. Yang, X.G., Li, L., Yan, X.J., Ding, L.: The structure and stability of pullback attractors for 3D Brinkman-Forchheimer equation with delay. Electron. Res. Arch. 28, 1395-1418 (2020)

24. Yang, Z.J., Li, Y.N.: Upper semicontinuity of pullback attractors for non-autonomous Kirchhoff wave equations. Discrete Contin. Dyn. Syst., Ser. B 24, 4899-4912 (2019)

25. Zelik, S.: Asymptotic regularity of solutions of a nonautonomous damped wave equation with a critical growth exponent. Commun. Pure Appl. Anal. 3, 921-934 (2004)

Submit your manuscript to a SpringerOpen ${ }^{\circ}$ journal and benefit from:

- Convenient online submission

- Rigorous peer review

Open access: articles freely available online

- High visibility within the field

- Retaining the copyright to your article

Submit your next manuscript at $\gg$ springeropen.com 